\title{
Encephaloduroarteriosynangiosis for hemorrhagic moyamoya disease: long-term outcome of a consecutive series of 95 adult patients from a single center
}

\author{
*Qian-Nan Wang, MD, PhD, Xiang-Yang Bao, MD, MS, Yong Zhang, MD, MS, Qian Zhang, MD, MS, \\ De-Sheng Li, MD, PhD, and Lian Duan, MD, PhD
}

Department of Neurosurgery, 307th Hospital, PLA Center for Cerebral Vascular Disease, Beijing, China

OBJECTIVE The objective of this study was to investigate long-term outcomes after encephaloduroarteriosynangiosis (EDAS) for the treatment of hemorrhagic moyamoya disease (MMD) and identify the risk factors for recurrent hemorrhages.

METHODS The authors retrospectively reviewed 95 patients with hemorrhagic MMD who were treated with EDAS at 307th Hospital PLA. Clinical features, angiographic findings, and clinical outcomes were investigated. Rebleeding incidences were compared between anterior or posterior hemorrhagic sites. Kaplan-Meier survival analysis and Cox proportional hazards regression models were used to estimate rebleeding risks after EDAS.

RESULTS The average age at symptom onset was 37.1 years (range $20-54$ years) for adult patients. The ratio of female to male patients was 1.16:1. In 61 of 95 hemorrhagic hemispheres (64.2\%), the anterior choroidal artery (AChA) or posterior communicating artery ( $\mathrm{PCOA}$ ) was extremely dilated, with extensive branches beyond the choroidal fissure, which only occurred in 28 of 86 nonhemorrhagic hemispheres (32.6\%). Fifty-seven incidences were classified as anterior hemorrhages and 38 as posterior. Sixteen of 95 patients $(16.8 \%)$ suffered cerebral rebleeding after a median follow-up duration of 8.5 years. The annual rebleeding rate was $2.2 \%$ per person per year. The incidence rate was higher for the posterior group than for the anterior group, but this difference was not statistically significant $(p>0.05)$. Cox regression analysis revealed that the age of symptom onset (OR 1.075, $95 \% \mathrm{Cl} 1.008-1.147, p=0.028$ ) was a predictor of rebleeding strokes.

CONCLUSIONS Through long-term follow up, EDAS proved beneficial for patients with hemorrhagic MMD. Dilation of the AChA-PCoA is associated with the initial hemorrhage of MMD, and rebleeding is age-related. Patients with hemorrhagic MMD should undergo follow-up over the course of their lives, even when neurological status is excellent.

https://thejns.org/doi/abs/10.3171/2017.12.JNS172246

KEYWORDS moyamoya disease; hemorrhage; encephaloduroarteriosynangiosis; long-term outcome; rebleeding; vascular disorders

$\mathrm{M}$ OYAMOYa disease (MMD) is a rare, chronic, and progressive cerebrovascular disorder that is characterized by stenosis and occlusion of the distal carotid, proximal middle, and anterior cerebral arteries and accompanied by small collateral vessel network development. ${ }^{31}$ This disease was first reported by Takeuchi and Shimizu in $1957^{34}$ and named moyamoya in 1969 by Su- zuki and Takaku, based on the angiographic appearance of collateral vessels. ${ }^{31}$ The etiological course of MMD remains unknown.

There are two main phenotypes of MMD in Asian populations, ischemic-type MMD (which is common in children) and hemorrhagic-type MMD (which is mostly observed in adults). ${ }^{7,30}$ According to Japanese guidelines

ABBREVIATIONS AChA = anterior choroidal artery; DSA = digital subtraction angiography; EDAS = encephaloduroarteriosynangiosis; ICA = internal carotid artery; ICH = intracranial hemorrhage; IVH = intraventricular hemorrhage; JAM = Japanese Adult Moyamoya; $M C A=$ middle cerebral artery; $M M D=$ moyamoya disease; $m R S=$ modified Rankin Scale; $\mathrm{PCI}=$ posterior circulation involvement; $\mathrm{PCOA}=$ posterior communicating artery; $\mathrm{SAH}=$ subarachnoid hemorrhage; $\mathrm{STA}=$ superficial temporal artery; $\mathrm{TIA}=$ transient ischemic attack.

SUBMITTED September 10, 2017. ACCEPTED December 11, 2017.

INCLUDE WHEN CITING Published online June 1, 2018; DOI: 10.3171/2017.12.JNS172246.

* Q.N.W. and X.Y.B. contributed equally to this work and share first authorship. 
regarding MMD treatment, surgical revascularization is recommended for ischemic-type MMD, which is further classified as either direct or indirect. ${ }^{27}$ Previous reports have demonstrated that surgical revascularization lowers the frequency of transient ischemic attacks (TIAs), reduces the risk of ischemic stroke recurrence, and greatly improves the prognosis for patients with ischemic-type MMD. ${ }^{20,23,29}$

However, there has been much controversy with respect to the outcomes of patients with hemorrhagic MMD who undergo surgery, and the feasibility of surgical treatment, surgical timing, selection of surgical procedures, and effect of surgical treatment in preventing rebleeding are subjects of debate. ${ }^{14,16}$ Studies have been designed to investigate surgery-associated problems, and specifically, the Japanese Adult Moyamoya (JAM) trial, ${ }^{24}$ a multicenter prospective study conducted at 22 high-volume centers across Japan, discovered that the risk of several events (including rebleeding, disabling stroke, and death) was more common in the medical group than in the direct revascularization group. The efficacy of indirect vascular reconstruction was not specifically addressed in this trial, and there are known differences in genetic background, clinical features, and therapeutic interventions related to MMD between China and other areas. To date, there has not been an investigation of revascularization for patients with hemorrhagic MMD in China.

Encephaloduroarteriosynangiosis (EDAS) is one of the most commonly used indirect vascular reconstruction methods. EDAS is considered easier and safer in patients with serious medical comorbidities, feasible in patients with inadequate recipient or donor artery grafts, $, 8,12,15,21,29$ and serves as a common procedure in our center. However, with regard to the efficacy of EDAS for hemorrhagic MMD, there is a paucity of multicenter long-term followup investigations across large sample sizes. In this paper we present a consecutive series of 95 adult patients with hemorrhagic MMD and detail our analysis of demographics, clinical characteristics, and long-term outcomes after EDAS. The goal of this study is to determine whether EDAS benefits patients with hemorrhagic MMD, analyze angiographic characteristics, and discover risk factors of recurrent hemorrhages.

\section{Methods}

\section{Patient Selection}

We identified all consecutive patients with MMD who were treated by EDAS at the Department of Neurosurgery of the 307th Hospital PLA, Beijing, China, from August 2003 through January 2010. Inclusion criteria included: 1) patient age $\geq 18$ years old; 2 ) patients diagnosed based on angiogram or MR angiography under guidelines of the Research Committee on MMD, according to the Ministry of Health and Welfare in Japan; and 3) patients who experienced at least 1 intracranial hemorrhage (ICH) that was verified by a CT scan, MRI, or lumbar puncture. The time interval between symptom presentation and imaging was $2.6 \pm 4.2$ hours (range 1.5-30.2 hours). As previously described, patients with unilateral MMD were included in the study. Exclusion criteria included the presence of a secondary moyamoya phenomenon caused by atherosclerosis, meningitis, Down syndrome, systemic vasculitis, hyperthyroidism, neurofibromatosis, leptospiral infection, or prior skull-base radiation therapy, and so on.

\section{Retrospective Chart Review}

Clinical records were reviewed, including hospital charts, clinic notes, and radiological studies. All data were collected through January 2017. The research ethics board at 307th Hospital approved the study design.

\section{Clinical Data}

The clinical data covered by our study include sex, onset age, hypertension, hyperlipidemia, diabetes mellitus, tobacco and alcohol use, preoperative angiographic stage, type of cerebral hemorrhage, posterior circulation involvement (PCI), and anterior or posterior location of cerebral hemorrhage. The preoperative angiographic stage was evaluated according to Suzuki's classification, and the higher Suzuki stage was used when 2 sides were different. The types of cerebral hemorrhage include ICH, intraventricular hemorrhage (IVH), subarachnoid hemorrhage (SAH), and IVH with ICH. The site of cerebral hemorrhage was judged in accordance with imaging-based classification criteria established by Takahashi et al. ${ }^{32}$ An anterior hemorrhage was defined as one attributable to perforating arteries from the anterior or middle cerebral artery (MCA), including those located in the putamen, caudate head, frontal lobe, anterior half of the temporal lobe, subependymal area of the anterior part of the lateral ventricle, or anterior half of the corpus callosum. A posterior hemorrhage was defined as one attributable to perforating arteries from the posterior cerebral artery or choroidal arteries, including those located in the thalamus, posterior half of the temporal lobe, parietal lobe, occipital lobe, subependymal area of the posterior part of the lateral ventricle including the trigon, or posterior half of the corpus callosum. Primary IVH, defined as IVH without intraparenchymal hemorrhage, was classified as either anterior or posterior according to the distribution of hematoma. Any diffusely distributed primary IVH whose origin was difficult to determine was classified as anterior. SAH without intracerebral hemorrhage was classified in a similar fashion.

\section{Angiographic Features}

The hemispheres were divided into a hemorrhagic group and nonhemorrhagic group, in accordance with CT findings. Each hemisphere was assessed for the association of angiographic changes of the anterior choroidal (AChA) and posterior communicating arteries (PCoAs) with hemorrhagic ictus. The AChA-PCoA were graded according to previously published methods: $: 18,25$ " 1 " refers to normal or slight-moderate dilation of AChA-PCoA with stenosed or occluded internal carotid artery (ICA) and proliferation around the circle of Willis; " 2 " refers to extreme dilation of AChA-PCoA with abnormal branches beyond the choroidal fissure, which serve as collateral blood supply to the anterior circulation via posterior pericallosal arteries and/or leptomeningeal collateral vessels; and " 3 " refers 
to nonvisualization of AChA-PCoA on digital subtraction angiography (DSA) with an occluded ICA proximal to the PCoA level.

\section{Surgical Treatment}

EDAS is the preferred surgical revascularization procedure at our institution. Briefly, EDAS involves placement of an external carotid artery branch beneath the dura in ischemic territories, and most commonly, we use the superficial temporal artery (STA). In certain circumstances, determined by the territory at risk, the occipital artery may also be used. Intraoperatively, the donor vessel with the strip of galea (the arterial bridge) is detached from the pericranium or the fascia below, and two burr holes are made beneath the proximal and distal ends of the arterial bridge. The burr holes are connected by milling to make an oval bone flap (in our cases the average size was $3.0 \times$ $8.0 \mathrm{~cm}$ ) and the dura is opened. The target artery is then sewn to the dura with a 10-0 Prolene suture. The bone flap is replaced after cutting out entry and exit sites for the envisaged artery. ${ }^{21}$

\section{Clinical Follow-Up}

Clinical follow-up evaluations were performed through clinical visits, telephone, or mail (by letter) interviews. DSA was obtained 6 months after EDAS. The development of collateral circulation of the MCA through bypass was graded according to the system described by Matsushima et al..20 grade A, in which the area supplied by surgical bypass covered more than two thirds of the MCA distribution; grade $\mathrm{B}$, in which one-third to two thirds of the MCA distribution was covered; and grade $\mathrm{C}$, in which only 1 cortical branch of the MCA was covered through the bypass, or no collateral circulation was observed. Improvement of the angiographic characteristics of AChA-PCoA was defined as reduction of dilation and branch extension of AChA-PCoA on follow-up DSA. Follow-up events included recurrent cerebral hemorrhage, cerebral infarction, and death, and cerebral hemorrhage and cerebral infarction were confirmed by CT or MRI. Postoperative stroke was defined as new neurological deficits lasting 24 hours or longer and associated with a new infarct or hemorrhage on MR or CT imaging in the first 30 days after the revascularization procedure. Evaluation of neurological outcome was assessed using a modified Rankin Scale (mRS). ${ }^{4} \mathrm{mRS}$ scores $\leq 2$ were defined as a good outcome and scores of 3 or more were defined as a poor outcome.

\section{Statistical Analysis}

All analyses were performed with the use of SPSS (version 20.0., IBM Corp.). Categorical variables were analyzed using the chi-square test, and continuous variables were compared using independent Student t-tests. Kaplan-Meier survival analysis and the Cox proportional hazards model were used to compare the incidences of recurrent hemorrhages in each subgroup, and analyze whether the site of cerebral hemorrhage functioned as a risk factor of rebleeding after EDAS. Other risk factors also included age, sex, hypertension, diabetes mellitus, hyperlipidemia,
TABLE 1. Baseline characteristics of patients

\begin{tabular}{cc}
\hline \multicolumn{1}{c}{ Variables } & Value \\
\hline Sex ratio (F/M) & $51: 44$ \\
\hline Mean age \pm SD (yrs) & $37.1 \pm 8.1$ \\
\hline Stroke risk factors, $\mathrm{n}(\%)$ & $16(16.8)$ \\
\hline Hypertension & $5(5.2)$ \\
\hline Diabetes mellitus & $2(2.1)$ \\
\hline Hyperlipidemia & $11(11.6)$ \\
\hline Smoking or drinking & \\
\hline Suzuki angiographic stage, $\mathrm{n}(\%)$ & $10(10.5)$ \\
\hline $\mathrm{II}$ & $16(16.8)$ \\
\hline $\mathrm{III}$ & $31(32.7)$ \\
\hline $\mathrm{IV}$ & $26(27.4)$ \\
\hline $\mathrm{V}$ & $12(12.6)$ \\
\hline $\mathrm{VI}$ & \\
\hline Type of hemorrhage, $\mathrm{n}(\%)$ & $40(42.1)$ \\
\hline $\mathrm{ICH}$ & $35(36.8)$ \\
\hline $\mathrm{IVH}$ & $12(12.6)$ \\
\hline $\mathrm{SAH}$ & $8(8.4)$ \\
\hline $\mathrm{IVH}$ w/ ICH & $20(21.1)$ \\
\hline $\mathrm{PCI}, \mathrm{n}(\%)$ & $9(9.5)$ \\
\hline Unilateral lesions, $\mathrm{n}(\%)$ & $57(60.0)$ \\
\hline Hemorrhagic site, $\mathrm{n}(\%)$ & $38(40.0)$ \\
\hline Anterior &
\end{tabular}

tobacco or alcohol use, PCI, and hemorrhage type. A probability value $<0.05$ was considered significant.

\section{Results}

\section{Demographics and Clinical Presentation}

A total of 95 adult patients with hemorrhagic MMD underwent EDAS in our hospital between August 2003 and January 2010. The average patient age at symptom onset was 37.1 years (range 20-54 years), and there were 51 female and 44 male patients (female/male ratio was 1.16:1; Table 1). Of the 95 patients with MMD, a history of hypertension was reported in 16 cases $(16.8 \%)$, history of diabetes in $5(5.2 \%)$, hyperlipidemia in $2(2.1 \%)$, and previous tobacco or alcohol use in $11(11.6 \%)$. The majority of patients presented with Suzuki angiographic stage IV or V (60.0\%), and 9 patients (9.5\%) displayed unilateral disease. Through CT scans, the presence of pure ICH was detected in 40 cases $(42.1 \%)$, pure IVH in $35(36.8 \%)$, SAH in 12 $(12.6 \%)$, and ICH with IVH in $8(8.4 \%)$. Twenty $(21.1 \%)$ of the 95 patients with hemorrhagic MMD underwent PCI.

The sites of cerebral hemorrhages for the 95 patients with hemorrhagic MMD were judged in accordance with image examination classification criteria for anterior/posterior hemorrhage established by Takahashi et al. ${ }^{32}$ Our results revealed that the number of patients with anterior hemorrhages was higher than those with posterior hemorrhages (57 cases, $60.0 \%$, vs 38 cases, $40.0 \%$; Table 1), however, this result does not display clear statistical sig- 
TABLE 2. Comparison of baseline characteristics and outcomes between the anterior and posterior groups

\begin{tabular}{cccc}
\hline Variable & Anterior $(\mathrm{n}=57)$ & Posterior $(\mathrm{n}=38)$ & p Value \\
\hline Mean age \pm SD $(\mathrm{yrs})$ & $36.5 \pm 6.9$ & $37.9 \pm 9.6$ & 0.398 \\
\hline Females, $\mathrm{n}(\%)$ & $26(45.6)$ & $25(65.8)$ & 0.053 \\
\hline Stroke risk factors, $\mathrm{n}(\%)$ & & $6(15.8)$ & 0.823 \\
\hline Hypertension & $10(17.5)$ & $1(2.6)$ & 0.348 \\
\hline Diabetes mellitus & $4(7.0)$ & 0 & 0.243 \\
\hline Hyperlipidemia & $2(3.5)$ & $4(10.5)$ & 0.793 \\
\hline Smoking or drinking & $7(12.3)$ & & 0.929 \\
\hline Types of hemorrhage, $\mathrm{n}(\%)$ & & $15(39.5)$ & $14(36.8)$ \\
\hline ICH & $25(43.9)$ & $5(13.2)$ & 0.173 \\
\hline IVH & $21(36.8)$ & $4(10.5)$ & 0.344 \\
\hline SAH & $7(12.3)$ & $7(18.4)$ & 0.748 \\
\hline IVH w/ ICH & $4(7.0)$ & & $8(21.1)$ \\
\hline PCl, $\mathrm{n}(\%)$ & $13(22.8)$ & $1(2.6)$ & $32(84.2)$ \\
\hline Follow-up events, $\mathrm{n}(\%)$ & $8(14.0)$ & 0 & \\
\hline Rebleeding & 0 & & \\
\hline Cerebral infarction & $45(78.9)$ & & \\
\hline mRS score 0-2, $\mathrm{n}(\%)$ & &
\end{tabular}

nificance. Baseline characteristics including age, sex, hypertension, diabetes mellitus, hyperlipidemia, tobacco or alcohol use, PCI, mRS scores, and hemorrhage type did not show statistically significant differences between anterior and posterior groups ( $p>0.05$; Table 2).

We also investigated angiographic dilation and extension of AChA-PCoA in 181 hemispheres. In 61 of the 95 hemorrhagic hemispheres (64.2\%), AChA-PCoA was dilated and showed branching (Grade 2), which occurred in 28 of 86 nonhemorrhagic hemispheres (32.6\%). The disappearance of AChA-PCoA due to occlusion of the ICA was observed in 18 of 95 hemorrhagic hemispheres (18.9\%), where only 4 cases presented nonhemorrhagic hemispheres $(4.7 \%)$; this difference was statistically significant $(\mathrm{p}<0.01)$.

\section{Follow-Up Arteriographic Findings}

Cerebral arteriographies were performed 6 months postoperatively to assess synangiosis efficacies and guide subsequent management. Although we requested followup arteriograms after 6 months from all patients, studies were refused by some families or could not be obtained in others due to economic or other considerations. Thirtyeight patients underwent postoperative cerebral arteriographies, and follow-up angiograms were performed after the EDAS operation in 74 hemispheres (mean 7.6 months, range 3.2-38 months). Twenty-seven percent of the 74 hemispheres investigated were classified as grade A collateral circulation, $47.3 \%$ as grade $\mathrm{B}$, and $25.7 \%$ as grade $\mathrm{C}$. Although we aspire to discover whether the development of collateral circulation after EDAS was associated with rebleeding, there was a paucity of imaging data for most patients at the time of rebleeding.

Improvements in AChA-PCoA extension were evaluated, in comparison with preoperative DSA, in 74 hemispheres from 38 patients. Seven patients (18.4\%) suffered cerebral rebleeding, and improvement in AChA-PCoA extension was observed in 51 of the 74 operated hemispheres (68.9\%). In hemorrhagic hemispheres, improvement in AChA-PCoA dilation and extension occurred in $28(90.3 \%)$ of the 31 patients who did not display rehemorrhage and in $3(42.9 \%)$ of the 7 patients who suffered rehemorrhage.

\section{Long-Term Clinical Outcome}

Patients were followed for an average duration of 8.5 \pm 1.7 years and experienced significant clinical improvements. However, 16 of the 95 patients $(16.8 \%)$ suffered cerebral rebleeding, and the annual rebleeding rate was $2.2 \%$ per person per year (Fig. 1). Three patients experienced TIAs after EDAS, and 1 patient suffered postoperative stroke after the revascularization procedure. Patients were assessed with regard to neurological respective outcomes, and 77 cases (81.1\%) achieved a postoperative mRS score $\leq 2$ points. Of the 16 patients who suffered rehemorrhages, 7 experienced no disabilities (mRS score 0 or 1), 2 suffered moderate disabilities (mRS score 3 ), and 7 died (mRS score 6) due to rebleeding.

In the anterior group, rebleeding was observed in 8 cases $(14.0 \%)$ and death in $5(8.8 \%)$, and none of the patients developed cerebral infarction. In the posterior hemorrhage group, rebleeding was observed in 8 cases $(21.1 \%)$, death in $2(5.3 \%)$, and 1 patient developed a cerebral infarction. The annual incidence rate of rebleeding was $1.6 \%$ per year for the anterior group and $3.1 \%$ per year for the posterior group. While the annual incidence rate of rebleeding was higher for the posterior group than for the anterior group, this difference was not statistically significant according to Kaplan-Meier analysis (Fig. 2; $\mathrm{p}=0.404$ for log-rank test).

Cox regression analysis of preoperative clinical variables revealed that older age at symptom onset (OR 1.075, 


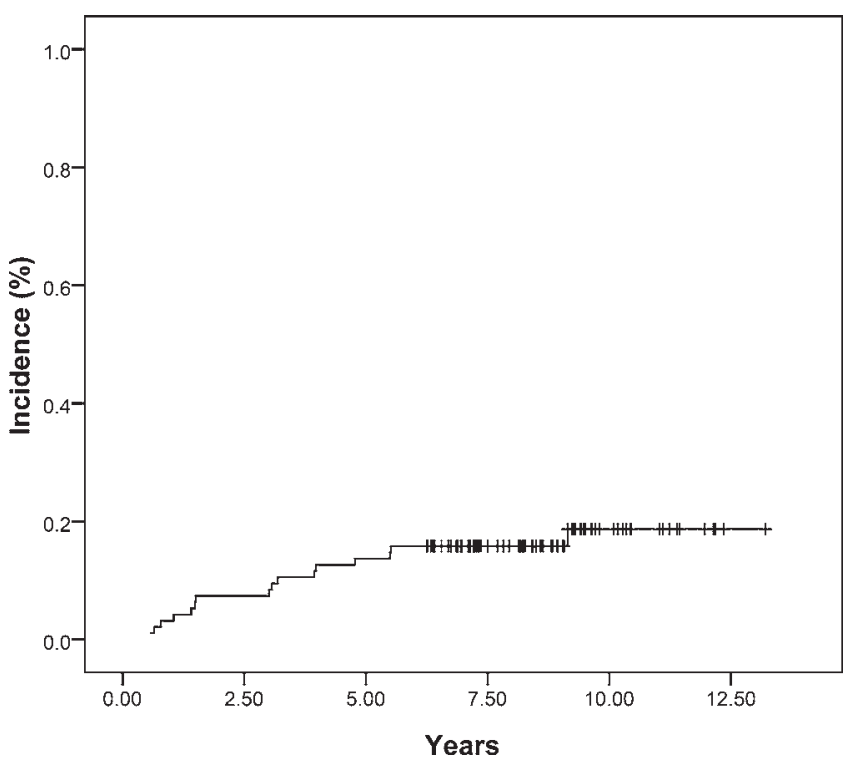

FIG. 1. Kaplan-Meier estimates of rebleeding incidence after surgery.

95\% CI $1.008-1.147 ; p=0.028$ ) was a positive predictor of rebleeding strokes. No significant correlations were observed between rebleeding and sex, hypertension, diabetes mellitus, hyperlipidemia, tobacco or alcohol use, hemorrhage type, hemorrhagic site, dilation grade of AChAPCoA in hemorrhagic hemispheres, unilateral lesions, or PCI (Table 3).

\section{Discussion}

MMD is a chronic vessel-occlusive cerebrovascular disorder with unclear etiological progression. With advancements in the development and optimization of clinical diagnostic technologies, the detection rate of MMD has gradually risen over recent years. Compared with ischemic-type MMD, hemorrhagic MMD shows higher morbidity and mortality rates and worse prognoses, and recently, hemorrhagic MMD has drawn more attention from neurosurgeons.

According to relevant epidemiological studies, Japan and East Asia are high incidence areas for hemorrhagic MMD, which mostly affects adults. ${ }^{3,10,22}$ Studies in 196 patients with MMD from 26 neurosurgical centers across Korea revealed that hemorrhagic MMD patients encompass $42.4 \%$ of all patients, with a higher rate $(69.0 \%)$ observed in adult populations. ${ }^{11}$ At present, therapeutic intervention for hemorrhagic MMD includes conservative medication and direct and indirect surgical revascularization, ${ }^{28}$ but there are disagreements pertaining to the most appropriate therapy. While numerous studies have indicated that surgical revascularization reduces rebleeding risk, there are substantially fewer investigations into the efficacy of indirect revascularization surgeries. In this study, the long-term follow-up of 95 adult patients with hemorrhagic MMD revealed a rebleeding rate of $16.8 \%$, a mean annual rebleeding rate of $2.2 \%$, and that age of symptom onset is a predictor of rebleeding stroke after EDAS treatment.

Currently, moyamoya vessels, arterial aneurysms, and ruptures of the AChA are the 3 main reasons for hem-

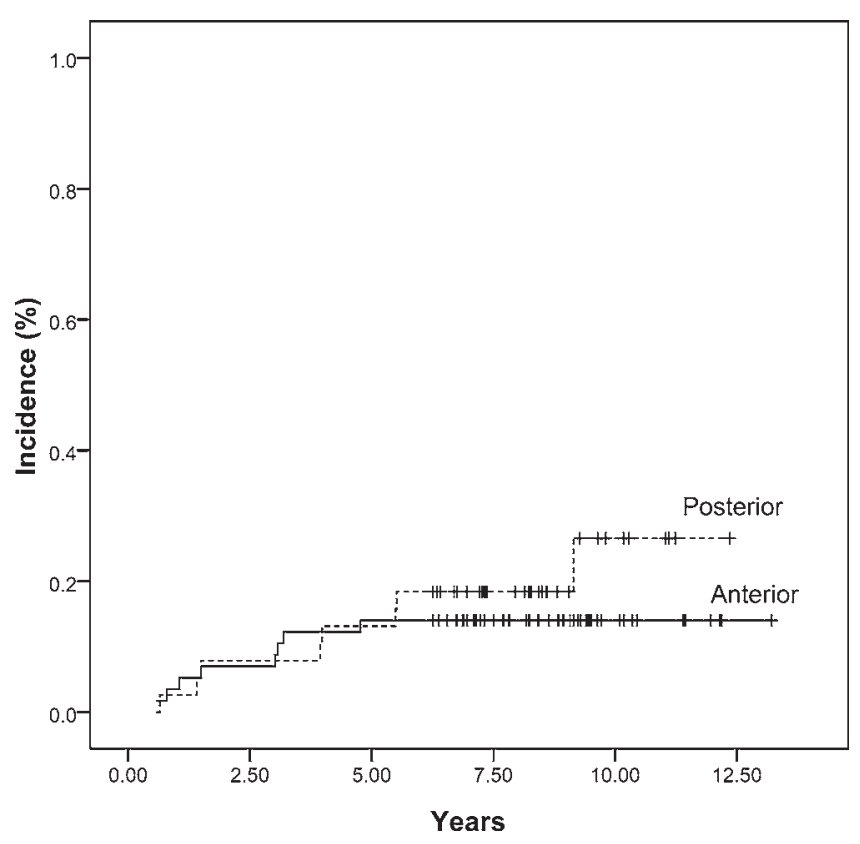

FIG. 2. Kaplan-Meier estimates of rebleeding incidence by hemorrhagic site.

orrhages in patients with MMD, ${ }^{18,33}$ and dilation of the AChA has attracted clinical attention. Similar findings were observed in our study through analyzing the dilation grade of the AChA-PCoA in 95 patients with hemorrhagic MMD. Morioka et al. analyzed AChA dilation in 107 patients with MMD and found that the risk of cerebral hemorrhages rises as the extent of AChA-PCoA dilation in the cerebral hemisphere displays increased severity. ${ }^{25}$ According to Morioka et al., the rupture of dilated AChAs and perforating arteries of the thalamus, rather than abnormal proliferation of small moyamoya vessels, is the main cause of hemorrhages in patients with MMD. Subsequent investigations by Liu et al. also discovered correlations between AChA-PCoA dilation and symptom onset in patients with MMD. ${ }^{17}$ We hypothesize that lateral ventricle hemorrhage is the main hemorrhage type in patients with MMD, and in

TABLE 3. Cox regression analyses for predictive factors of postoperative hemorrhage events

\begin{tabular}{lcccc}
\hline \multicolumn{1}{c}{ Characteristic } & $\mathrm{p}$ Value & OR & \multicolumn{2}{c}{$95 \% \mathrm{Cl}$} \\
\hline Female & 0.266 & 1.881 & 0.618 & 5.722 \\
\hline Age at onset & 0.028 & 1.075 & 1.008 & 1.147 \\
\hline Type of hemorrhage & 0.571 & 1.179 & 0.668 & 2.082 \\
\hline Hemorrhagic site & 0.734 & 1.193 & 0.431 & 3.297 \\
\hline Hypertension & 0.221 & 3.752 & 0.454 & 31.007 \\
\hline Diabetes mellitus & 0.447 & 0.427 & 0.048 & 3.831 \\
\hline Hyperlipidemia & 0.987 & 0 & 0 & 0 \\
\hline Smoking or drinking & 0.805 & 1.337 & 0.132 & 13.501 \\
\hline Dilation of AChA-PCoA & 0.458 & 0.605 & 0.247 & 1.483 \\
\hline Unilateral lesions & 0.540 & 0.487 & 0.049 & 4.870 \\
\hline PCl & 0.132 & 2.386 & 0.769 & 7.406 \\
\hline
\end{tabular}


support of our theory, we observed that the lateral ventricle choroid plexus artery comprised the AChA branch of the ICA, the branch of the posteromedial choroidal artery, and the posterolateral choroidal artery branch of the posterior cerebral artery. Because stenosis and occlusion of the distal carotid is observed in patients with MMD, compensatory enlargement frequently appears in the $\mathrm{AChA}$, and as the dilated AChA suffers more stress, the risk of hemorrhage increases.

Thirty-eight patients underwent follow-up angiograms, which allowed us to compare improvements in AChAPCoA extension with preoperative DSA. Fifty-one of 74 hemispheres (68.9\%) displayed improvement in AChAPCoA extension after EDAS, which suggests that EDAS can relieve AChA-PCoA dilation in patients with hemorrhagic MMD. We speculate that surgery improves local hemodynamics and the formation of collateral vessels lightens stress on vessels in the AChA, which together reduce rebleeding risk. We also compared angiographic changes of the AChA-PCoA in hemorrhagic hemispheres between patients who suffered rebleeding in surgical hemispheres and those who did not, and interestingly, patients who do not suffer rehemorrhages tend to have more frequent improvements in AChA-PCoA extension.

Cerebral rebleeding was the most significant malady to influence the prognosis of patients with hemorrhagic MMD, and investigations into the cause of cerebral rebleeding are of utmost importance. In our study, 16 patients suffered cerebral rebleeding (16.8\%), 7 of whom died due to rebleeding. Kobayashi et al. studied 42 patients with hemorrhagic MMD who had received conservative medication over a mean follow-up interval of 6.7 years, ${ }^{16}$ and discovered that the effects of conservative medication were poor with respect to ameliorating rebleeding rates $(33.3 \%)$, as the annual rebleeding rate was $7.09 \%$ in men. Research of Liu et al. at Beijing Tiantan Hospital found that the rebleeding rate after surgical revascularization was $7.4 \%$, and patients benefit more from intervention surgery than conservative medication. ${ }^{19}$ In 1990 , a multicenter, prospective, and randomized controlled trial was conducted across 22 clinical centers in Japan to investigate the effect of revascularization on 80 adult patients with hemorrhagic MMD. This trial revealed that the rebleeding rate of patients who underwent surgical revascularization was $14.3 \%$, while that of patients who received conservative medication was $34.2 \%$, which directly demonstrated that in comparison with conservative medication, the risk of rebleeding is significantly reduced by direct revascularization. ${ }^{24}$ In further agreement, Amin-Hanjani et al. reported that in patients with hemorrhagic MMD, rebleeding risk could be reduced from $30 \%-65 \%$ to $12.5 \%-20 \%$ by direct revascularization. ${ }^{1}$

However, Houkin et al. reported a lack of a difference between direct and indirect revascularization in preventing rebleeding for patients with MMD, ${ }^{9}$ while Kawaguchi et al. showed that direct revascularization prevents rebleeding better than EDAS. ${ }^{13}$ Our long-term follow-up of Chinese patients who underwent EDAS revealed a rebleeding rate of $16.8 \%$, which was lower than in patients who received conservative medication in Beijing Tiantan Hospital and agrees with the results of many other stud- ies in Japan and South Korea that investigated rebleeding rates in patients who underwent direct revascularization. Due to epidemiological differences in the onset of MMD, we could not perform prospective and randomized controlled trials to investigate the effects of revascularization for patients with MMD. Our study also lacked unified principles and standards, and our center will next conduct a prospective and randomized controlled trial to determine the effects of revascularization for Chinese patients with hemorrhagic MMD. It is worth noting that a recent published study from the JAM group found an association between choroidal anastomosis and rebleeding. ${ }^{6}$ Funaki et al. revealed that choroidal anastomosis and PCA involvement are characteristic of posterior hemorrhage in MMD, and choroidal anastomosis might be considered a potential source of posterior hemorrhage at high risk of rebleeding. These conclusions are of great significance and will guide future randomized controlled trials in our center.

Takahashi et al. divided patients into posterior and anterior cerebral hemorrhage groups, based on the results of a Japanese adult MMD trial, to analyze rebleeding incidence differences at distinct primary hemorrhage sites. ${ }^{32}$ The results of this study revealed that the rebleeding rate was higher for the posterior group than for the anterior group. In our study, we divided 95 patients who underwent EDAS into posterior cerebral hemorrhage and anterior cerebral hemorrhage groups, in terms of the division criterion by Takahashi et al., and consistent with his results found that the annual rebleeding rate was higher for the posterior group than for the anterior group. However, this difference was not statistically significant. According to Morioka et al., ${ }^{25}$ rupture of the dilated AChA leads to hemorrhage in lateral ventricles and rupture of the dilated branch of the PCoA results in hemorrhage in the thalamus. These bleeding sites are located in the posterior cerebrum, which causes a higher rebleeding risk for the posterior cerebrum than for the anterior cerebrum.

Cox survival analysis revealed that age of symptom onset is a positive predictor of rebleeding strokes, and the risk of cerebral rebleeding rises with increasing age. Morioka et al. reported rebleeding peak periods when pediatric patients with MMD grow older, especially during the age interval of 46-55 years. ${ }^{26}$ Also, other studies have found that the rebleeding risk of patients with hemorrhagic MMD increases with prolonged follow-up duration. ${ }^{2,5,13,35}$ We speculate that with increased age, small blood vessels gradually become blocked, vessel brittleness increases, pressure of blood flow in moyamoya vessels rises, and the risk of blood vessel rupture increases. Therefore, from the results of our study and previous investigations, we suggest that patients with MMD who are of older age at the time of hemorrhage symptom onset actively participate in clinical follow-ups and frequent imaging examinations, even in spite of neurological recovery after treatment with medicine or surgery. Furthermore, the Cox proportional hazards model suggested that dilation of AChA-PCoA in hemorrhagic hemiencephalon was not the primary rebleeding risk factor for patients treated with EDAS.

\section{Limitations}

Our study has some limitations. First, a control group 
was not set, so we could not accurately account for differences between the effects of EDAS, conservative treatments, and direct surgical revascularizations. Second, our study was retrospectively conducted at a single center, and case selection was not randomized and included a patient population that lacked heterogeneity, which influences the grouping of results for posterior and anterior cerebral hemorrhages. Third, fewer than $50 \%$ of the patients underwent postoperative angiograms at the 6-month follow-up, and the size of this sample is insufficient to enable firm conclusions to be drawn regarding the effect of EDAS on collateralization or angiographic outcomes. In addition, we did not acquire imaging data when rebleeding occurred, and as such, we could not explore whether a correlation exists between collateral compensative situations and cerebrum rebleeding after EDAS.

\section{Conclusions}

Dilation of the AChA is closely related to the onset of cerebral hemorrhage symptoms. Rebleeding rates were higher for patients with posterior hemorrhages than for those with anterior hemorrhages, and the risk of rebleeding for patients with MMD heightens with increased age of symptom onset. Patients with MMD who are of older age at hemorrhage symptom onset should actively participate in clinical follow-ups.

\section{Acknowledgments}

This study was supported by a grant from the National Nature Science Foundation of China (no. 81571136) and the Beijing Municipal Science \& Technology Commission (no. Z171100001017144).

\section{References}

1. Amin-Hanjani S, Barker FG II, Charbel FT, Connolly ES Jr, Morcos JJ, Thompson BG: Extracranial-intracranial bypass for stroke-is this the end of the line or a bump in the road? Neurosurgery 71:557-561, 2012

2. Aoki N: Cerebrovascular bypass surgery for the treatment of Moyamoya disease: unsatisfactory outcome in the patients presenting with intracranial hemorrhage. Surg Neurol 40:372-377, 1993

3. Baba T, Houkin K, Kuroda S: Novel epidemiological features of moyamoya disease. J Neurol Neurosurg Psychiatry 79:900-904, 2008

4. Bamford JM, Sandercock PA, Warlow CP, Slattery J: Interobserver agreement for the assessment of handicap in stroke patients. Stroke 20:828, 1989

5. Choi JU, Kim DS, Kim EY, Lee KC: Natural history of moyamoya disease: comparison of activity of daily living in surgery and non surgery groups. Clin Neurol Neurosurg 99 (Suppl 2):S11-S18, 1997

6. Funaki T, Takahashi JC, Houkin K, Kuroda S, Takeuchi S, Fujimura M, et al: Angiographic features of hemorrhagic moyamoya disease with high recurrence risk: a supplementary analysis of the Japan Adult Moyamoya Trial. J Neurosurg [epub ahead of print April 14, 2017; DOI: 10.3171/2016.11. JNS161650]

7. Han DH, Kwon OK, Byun BJ, Choi BY, Choi CW, Choi JU, et al: A co-operative study: clinical characteristics of 334 Korean patients with moyamoya disease treated at neurosurgical institutes (1976-1994). Acta Neurochir (Wien) 142:1263-1274, 2000
8. Houkin K, Ishikawa T, Yoshimoto T, Abe H: Direct and indirect revascularization for moyamoya disease surgical techniques and peri-operative complications. Clin Neurol Neurosurg 99 (Suppl 2):S142-S145, 1997

9. Houkin K, Kamiyama H, Abe H, Takahashi A, Kuroda S: Surgical therapy for adult moyamoya disease. Can surgical revascularization prevent the recurrence of intracerebral hemorrhage? Stroke 27:1342-1346, 1996

10. Hung CC, Tu YK, Su CF, Lin LS, Shih CJ: Epidemiological study of moyamoya disease in Taiwan. Clin Neurol Neurosurg 99 (Suppl 2):S23-S25, 1997

11. Ikezaki K, Han DH, Kawano T, Kinukawa N, Fukui M: A clinical comparison of definite moyamoya disease between South Korea and Japan. Stroke 28:2513-2517, 1997

12. Ishikawa T, Houkin K, Kamiyama H, Abe H: Effects of surgical revascularization on outcome of patients with pediatric moyamoya disease. Stroke 28:1170-1173, 1997

13. Kawaguchi S, Okuno S, Sakaki T: Effect of direct arterial bypass on the prevention of future stroke in patients with the hemorrhagic variety of moyamoya disease. J Neurosurg 93:397-401, 2000

14. Kikuta K, Takagi Y, Nozaki K, Sawamoto N, Fukuyama H, Hashimoto N: The presence of multiple microbleeds as a predictor of subsequent cerebral hemorrhage in patients with moyamoya disease. Neurosurgery 62:104-112, 2008

15. Kim SK, Cho BK, Phi JH, Lee JY, Chae JH, Kim KJ, et al: Pediatric moyamoya disease: An analysis of 410 consecutive cases. Ann Neurol 68:92-101, 2010

16. Kobayashi E, Saeki N, Oishi H, Hirai S, Yamaura A: Longterm natural history of hemorrhagic moyamoya disease in 42 patients. J Neurosurg 93:976-980, 2000

17. Liu P, Liu AH, Han C, Chen C, Lv XL, Li DS, et al: Difference in angiographic characteristics between hemorrhagic and nonhemorrhagic hemispheres associated with hemorrhage risk of moyamoya disease in adults: a self-controlled study. World Neurosurg 95:348-356, 2016

18. Liu W, Zhu S, Wang X, Yue X, Zhou Z, Wang H, et al: Evaluation of angiographic changes of the anterior choroidal and posterior communicating arteries for predicting cerebrovascular lesions in adult moyamoya disease. J Clin Neurosci 18:374-378, 2011

19. Liu X, Zhang D, Shuo W, Zhao Y, Wang R, Zhao J: Long term outcome after conservative and surgical treatment of haemorrhagic moyamoya disease. J Neurol Neurosurg Psychiatry 84:258-265, 2013

20. Matsushima T, Inoue T, Suzuki SO, Fujii K, Fukui M, Hasuo $\mathrm{K}$ : Surgical treatment of moyamoya disease in pediatric patients-comparison between the results of indirect and direct revascularization procedures. Neurosurgery 31:401-405, 1992

21. Matsushima Y, Aoyagi M, Suzuki R, Tabata H, Ohno K: Perioperative complications of encephalo-duro-arterio-synangiosis: prevention and treatment. Surg Neurol 36:343-353, 1991

22. Matsushima Y, Qian L, Aoyagi M: Comparison of moyamoya disease in Japan and moyamoya disease (or syndrome) in the People's Republic of China. Clin Neurol Neurosurg 99 (Suppl 2):S19-S22, 1997

23. Mesiwala AH, Sviri G, Fatemi N, Britz GW, Newell DW: Long-term outcome of superficial temporal artery-middle cerebral artery bypass for patients with moyamoya disease in the US. Neurosurg Focus 24(2):E15, 2008

24. Miyamoto S, Yoshimoto T, Hashimoto N, Okada Y, Tsuji I, Tominaga T, et al: Effects of extracranial-intracranial bypass for patients with hemorrhagic moyamoya disease: results of the Japan Adult Moyamoya Trial. Stroke 45:1415-1421, 2014

25. Morioka M, Hamada J, Kawano T, Todaka T, Yano S, Kai Y, et al: Angiographic dilatation and branch extension of the anterior choroidal and posterior communicating arteries are 
predictors of hemorrhage in adult moyamoya patients. Stroke 34:90-95, 2003

26. Morioka M, Hamada J, Todaka T, Yano S, Kai Y, Ushio Y: High-risk age for rebleeding in patients with hemorrhagic moyamoya disease: long-term follow-up study. Neurosurgery 52:1049-1055, 2003

27. Hashimoto N: Guidelines for diagnosis and treatment of moyamoya disease (spontaneous occlusion of the circle of Willis). Neurol Med Chir (Tokyo) 52:245-266, 2012

28. Ryan RW, Chowdhary A, Britz GW: Hemorrhage and risk of further hemorrhagic strokes following cerebral revascularization in moyamoya disease: a review of the literature. Surg Neurol Int 3:72, 2012

29. Scott RM, Smith JL, Robertson RL, Madsen JR, Soriano SG, Rockoff MA: Long-term outcome in children with moyamoya syndrome after cranial revascularization by pial synangiosis. J Neurosurg 100 (2 Suppl Pediatrics):142-149, 2004

30. Suzuki J, Kodama N: Moyamoya disease-a review. Stroke 14:104-109, 1983

31. Suzuki J, Takaku A: Cerebrovascular "moyamoya" disease. Disease showing abnormal net-like vessels in base of brain. Arch Neurol 20:288-299, 1969

32. Takahashi JC, Funaki T, Houkin K, Inoue T, Ogasawara K, Nakagawara J, et al: Significance of the hemorrhagic site for recurrent bleeding: prespecifed analysis in the Japan Adult Moyamoya Trial. Stroke 47:37-43, 2016

33. Takahashi JC, Miyamoto S: Moyamoya disease: recent progress and outlook. Neurol Med Chir (Tokyo) 50:824-832, 2010
34. Takeuchi K, Shimizu K: Hypogenesis of bilateral internal carotid arteries. No To Shinkei 9:37-43, 1957

35. Yoshida Y, Yoshimoto T, Shirane R, Sakurai Y: Clinical course, surgical management, and long-term outcome of moyamoya patients with rebleeding after an episode of intracerebral hemorrhage: an extensive follow-up study. Stroke 30:2272-2276, 1999

\section{Disclosures}

The authors report no conflict of interest concerning the materials or methods used in this study or the findings specified in this paper.

\section{Author Contributions}

Conception and design: Duan. Acquisition of data: Wang, Bao, Y Zhang. Analysis and interpretation of data: Wang, Bao, Y Zhang. Drafting the article: Wang, Bao. Critically revising the article: Duan. Reviewed submitted version of manuscript: Duan. Approved the final version of the manuscript on behalf of all authors: Duan. Statistical analysis: Wang, Bao. Administrative/ technical/material support: Wang, Bao, Q Zhang. Study supervision: Duan, Li.

\section{Correspondence}

Lian Duan: 307th Hospital, PLA Center for Cerebral Vascular Disease, Beijing, China. duanlian307@sina.com. 